\title{
FERTIRRIGAÇÃO COM DIFERENTES DOSES DE NPK E SEUS EFEITOS SOBRE A PRODUÇÃO E QUALIDADE DE FRUTOS DE LARANJA (Citrus sinensis O.) 'VALÊNCIA'
}

\author{
LUIZA HELENA DUENHAS², ROBERTO LYRA VILLAS BÔAS ${ }^{3}$, CLÁUDIO MÁRCIO PEREIRA DE \\ SOUZA $^{4}$, CARLOS RENATO ALVES RAGOZO ${ }^{5}$, LEONARDO THEODORO BULL ${ }^{3}$
}

\begin{abstract}
RESUMO - O experimento foi realizado na Fazenda Três Irmãos, no município de Pratânia, Estado de São Paulo, com o objetivo de estudar o efeito da fertirrigação com diferentes doses de NPK aplicadas por meio de um sistema de irrigação por microaspersão na cultura da laranja em comparação com a adubação convencional irrigada e não irrigada. A avaliação dos efeitos da irrigação e da fertirrigação na cultura foi feita com base na produção e qualidade dos frutos colhidos, na análise de solo da camada de 0-20 cm e na análise de folhas. Os tratamentos não influenciaram significativamente a produtividade de acordo com a análise estatística realizada, embora se tenha observado uma tendência de maior produtividade nos tratamentos de fertirrigação para uma mesma dose. A análise de qualidade dos frutos não mostrou diferenças significativas entre os tratamentos. Os teores de alguns dos elementos obtidos na análise de solo apresentaram diferenças significativas proporcionadas pela irrigação e/ou modo de aplicação dos fertilizantes à cultura. A análise de folhas diferiu quanto aos teores de $\mathrm{Ca}, \mathrm{Mg}$ e $\mathrm{K}$.
\end{abstract}

Termos para indexação: irrigação, fertirrigação, laranja.

\section{FERTIGATION WITH DIFFERENT DOSES OF NPK AND ITS EFFECT ON FRUIT YIELD AND QUALITY OF VALENCIA ORANGE (Citrus sinensis Osbeck).}

\begin{abstract}
The experiment was carried out in Fazenda Três Irmãos, in the municipal district of Pratânia, State of São Paulo, with the objective of studying the effect of fertigation with different rates of applied NPK through a microsprinkler irrigation system for an orange crop in comparison with conventional fertilization with and without irrigation. The evaluation of the irrigation and fertigation effects on the crop was done considering the production and quality of the fruits, the $0-20 \mathrm{~cm}$ layer soil analysis and the leaves analyzes. The treatments did not influence the productivity significantly, although a tendency of larger productivity was observed for the fertigation treatments. The quality of the fruits was also not affected. The results of soil analysis indicated differences for some of the elements due to the irrigation either/or way of fertilizers application. The leaves analyses differed as the $\mathrm{Ca}, \mathrm{Mg}$ and $\mathrm{K}$ tenors.
\end{abstract}

Index terms: irrigation, fertigation, orange.

\section{INTRODUÇÃO}

A utilização da irrigação em pomares de citros vem experimentando grande incremento, em virtude dos bons resultados que propicia, particularmente: elevação da produtividade dos pomares, melhoria da qualidade das frutas e a possibilidade de produção fora de época.

A fertirrigação consiste na aplicação dos adubos juntamente com a água de irrigação. Tal operação, além de ser de grande utilidade para as plantas, pois o nutriente é fornecido juntamente com a água (essencial para sua absorção), apresenta ainda muitas outras vantagens, entre as quais a de melhor distribuição do fertilizante no campo e a possibilidade de maior parcelamento das adubações, aumentando a eficiência na utilização dos adubos pelas plantas.

Apesar de existirem publicações sobre a aplicação de fertilizantes via água de irrigação, constata-se que ainda há necessidade de pesquisa sobre doses, concentração e parcelamento de fertilizantes para as culturas, uma vez que existem muitas variáveis envolvidas no emprego dessa técnica.

O presente trabalho tem o objetivo de estudar os efeitos da fertirrigação com diferentes doses de NPK sobre a produção e a qualidade dos frutos de laranja-Valência quando comparados à aplicação convencional de fertilizantes.

\section{MATERIAL E MÉTODOS}

O experimento foi realizado no município de Pratânia, Estado de São Paulo, em uma área de produção comercial de laranja-Valência, utilizando como porta-enxerto a tangerinaCleópatra. O pomar apresentava espaçamento de 7 metros entre linhas e 4 metros entre plantas, e idade de 6 anos.

A irrigação, assim como a fertirrigação, foi iniciada quando o pomar apresentava 5 anos de idade. Este trabalho referese ao segundo ano de aplicação dos tratamentos.

1 (Trabalho 076/2001). Recebido: 27/03/2001. Aceito para publicação: 01/02/2002.

2 Engenheira Agrônoma, Ms, doutoranda, LER - ESALQ/USP. Av. Pádua Dias,11, 13418-900, Piracicaba-SP. E-mail: lduenhas@esalq.usp.br

3 Professor do Depto de Recursos Naturais, FCA/Unesp, Botucatu-SP

4 Engenheiro Agrícola, Ms, doutorando do Depto. de Engenharia Rural, FCA/Unesp, Botucatu-SP

5 Engenheiro Agrônomo, Ms, CATI/Pratânia-SP 
O solo apresenta textura arenosa, classificando-se em Neossolo quartzarênico, segundo a EMBRAPA (1999).

A partir dos resultados das análises de folhas e solo, foram estabelecidas as doses de fertilizantes segundo Quaggio et al. (1997), que foram de $200 \mathrm{~kg} \cdot \mathrm{ha}^{-1} \mathrm{de} \mathrm{N} ; 60 \mathrm{~kg} \cdot \mathrm{ha}^{-1}$ de $\mathrm{P}_{2} \mathrm{O}_{5} ; 100$ $\mathrm{kg} . \mathrm{ha}^{-1} \mathrm{~K}_{2} \mathrm{O}$ e $2 \mathrm{~kg} \cdot \mathrm{ha}^{-1}$ de B. Os fertilizantes utilizados foram: uréia $(45 \% \mathrm{~N})$; cloreto de potássio branco $\left(60 \% \mathrm{~K}_{2} \mathrm{O}\right)$; fosfato monoamônico purificado $\left(60 \% \mathrm{P}_{2} \mathrm{O}_{5}\right.$ e $\left.10 \% \mathrm{~N}\right)$ e ácido bórico (17\% B). Via foliar, foram utilizados Nutrimins Zn (7\% Zn e 3\% S) e Nutrimins Mn (7\% Mn e 3\% S).

Para a realização da irrigação, foi instalado um sistema de irrigação por microaspersão, utilizando emissores autocompensantes. Utilizou-se um emissor por planta, localizado na posição média entre duas plantas. $\mathrm{O}$ manejo da irrigação baseou-se na utilização do Tanque Classe A.

O delineamento utilizado foi em blocos casualizados, com cinco tratamentos e oito repetições. Os tratamentos foram os seguintes: (SI) adubação convencional sem irrigação; (CI) adubação convencional irrigada; (FI) fertirrigação com dose completa; (F1/2) fertirrigação com metade da dose; (F1/3) fertirrigação com um terço da dose.

Os tratamentos referentes à adubação convencional tiveram a dose anual de $\mathrm{NPK}+\mathrm{B}$ na forma sólida parcelada em três vezes: $40 \%$ em outubro, $30 \%$ em dezembro e $30 \%$ em fevereiro de 1999, sendo o fertilizante aplicado na projeção da copa. Os tratamentos utilizando a fertirrigação tiveram suas doses divididas em dez vezes iguais, com uma aplicação mensal a partir de setembro de 1998 e com a última aplicação em junho de 1999. A colheita foi realizada em agosto de 1999, sendo a produção analisada considerando peso e número de frutos e produção por hectare.

Dos frutos colhidos foram tomadas amostras aleatórias de 20 frutos por parcela, avaliando-se: espessura de casca, acidez total titulável (Pregnolatto \& Pregnolatto, 1985), teor de sólidos solúveis totais (Tressler \& Joslyn, 1961), relação entre sólidos solúveis totais e acidez total titulável ou "ratio" (Tressler \& Joslyn, 1961), altura e diâmetro de frutos, peso médio de frutos e rendimento de suco.

Foi realizada coleta de amostras de solo na camada de 0 $-20 \mathrm{~cm}$, em agosto de 1999. A coleta de folhas foi realizada em março de 1999, sendo que a amostragem das folhas seguiu as recomendações do Grupo Paulista de Adubação e Calagem em Cítrus (1994).

\section{RESULTADOS E DISCUSSÃO}

\section{Parâmetros de produção}

Para as condições em que foi realizado este experimento, não foram encontradas diferenças significativas para os parâmetros: peso de fruto, número de frutos por planta e produtividade. No entanto, é possível notar determinadas tendências quanto aos três parâmetros, apresentados na Tabela 1 .

Considerando que os tratamentos SI, CI e FI receberam a mesma quantidade de fertilizantes durante o ciclo, observa-se que o tratamento FI apresentou maior produtividade que os tratamentos CI e SI, respectivamente. Quanto aos tratamentos que utilizaram fertirrigação, nota-se que a produtividade esteve positivamente correlacionada à quantidade de fertilizante aplicado.

A diferença entre os tratamentos, para o parâmetro produtividade, não foi significativa. Isto pode ser explicado pelo pouco tempo de aplicação dos tratamentos, que pode ter sido insuficiente para produzir diferenças significativas. Outro fator a ser considerado, é o parcelamento adotado. Um maior parcelamento poderia resultar em um efeito mais significativo sobre a produção, principalmente devido a uma maior eficiência de aproveitamento do nitrogênio.

Da mesma forma, Tomlinson \& Coetzee (1997) não obtiveram diferenças de produtividade quando compararam aplicação convencional de fertilizantes e várias freqüências de fertirrigação em laranja Midnight Valência.

Os resultados divergem dos obtidos por Alva et al. (1998), que obtiveram diferenças significativas de produtividade em laranja-Valência quando compararam aplicação convencional de $\mathrm{N}$ e fertirrigação por um período de dois anos. A produtividade obtida com a fertirrigação foi superior.

Para as condições em que foi realizado este experimento, a produtividade foi influenciada em maior parte pelo número de frutos por planta do que pelo peso médio de frutos. A correlação entre os parâmetros produtividade e número de frutos por ha apresentou $\mathrm{r}^{2}=0,828$. Guardiola (2000) afirma que o número de frutos por planta está relacionado de modo direto com a produtividade final em citros.

O peso individual dos frutos superou a média da variedade, que, segundo Figueiredo (1991), é de $150 \mathrm{~g}$, sendo a produção destinada ao mercado externo de consumo 'in natura'.

\section{Parâmetros de qualidade de fruto}

Foram analisados os seguintes parâmetros de qualidade dos frutos: acidez total titulável (expressa em \%), sólidos solúveis totais (brix), 'ratio', espessura de casca (mm), rendimento de suco (\%), diâmetro e altura do fruto $(\mathrm{mm})$. Os parâmetros não apresentaram diferença significativa para os tratamentos estudados (Tabela 2). Os resultados estão de acordo com Alva \& Paramasivam (1998), que afirmaram que a variação entre níveis de nitrogênio não afeta os parâmetros de qualidade do suco obtido dos frutos cítricos nem o tamanho destes.

Intrigliolo et al. (1999) não obtiveram diferença entre os parâmetros de qualidade de suco quando compararam aplicação convencional de fertilizantes e fertirrigação em pomar de laranjaValência Late, apesar de terem observado menor peso de frutos na aplicação convencional de fertilizantes. Dasberg et al. (1988) não encontraram diferenças na qualidade e tamanho de frutos quando compararam aplicação convencional de fertilizantes e fertirrigação, utilizando diferentes doses de nitrogênio.

Para o parâmetro espessura de casca, foi calculado o contraste para comparar os tratamentos SI e CI, sendo significativo a 2,5\%, apontando para efeito da irrigação. Este resultado confirma o obtido por Castel (1994), que observou redução da espessura de casca com aumento nos níveis de irrigação.

Algumas diferenças, quando os parâmetros de qualidade são comparados aos padrões da variedade, se devem à antecipação da colheita. Os valores de acidez obtidos para os tratamentos são considerados altos para laranja-Valência, que, segundo Figueiredo (1991), são de 1,05\%. O valor médio de sólidos solúveis totais (SST) da variedade é de $11,8^{\circ}$ Brix. Desta forma, 
os valores obtidos para 'ratio', que é a relação entre SST e acidez, estão abaixo da média, que é de 11,2 . O rendimento de suco obtido para todos os tratamentos está abaixo do padrão, que, segundo Figueiredo (1991), é de 50\%, em média.

\section{Análise química de solo}

A média dos tratamentos quanto à análise química do solo está representada na Tabela 3.

Os tratamentos diferiram quanto ao $\mathrm{pH}$, segundo a análise de variância. $\mathrm{O}$ contraste entre os tratamentos de fertirrigação e os tratamentos de adubação convencional, para o $\mathrm{pH}$, foi significativo, sendo que também foi significativo o contraste entre o tratamento convencional sem irrigação e o convencional irrigado. Desta forma, pode-se reafirmar que o modo de aplicação de fertilizantes e a irrigação interferem no comportamento do $\mathrm{pH}$ do solo.

Uma das hipóteses para explicar a diferença entre os tratamentos irrigado e não irrigado é que, neste último, houve menor lixiviação de bases e, conseqüentemente, o abaixamento de $\mathrm{pH}$ foi menor.

Considerando os tratamentos de fertirrigação, pode-se notar que aqueles com maior dose de NPK (FI e F1/2) apresentaram maior acidificação do solo que o tratamento com menor dose de NPK (F1/3), lembrando que duas das fontes utilizadas reagem de forma ácida no solo: a uréia e o MAP.

Observando-se os valores obtidos no solo para $\mathrm{Ca}$ trocável, nota-se que os tratamentos que obtiveram maiores valores também foram aqueles que alcançaram os maiores valores de $\mathrm{pH}$. O mesmo comportamento pode ser observado para os valores de Mg. Segundo Haynes \& Swift (1987), a acidificação do solo na região atingida pela irrigação resulta em significativa redução nos teores de $\mathrm{Ca}, \mathrm{Mg}$ e $\mathrm{K}$ extraídos na análise do solo amostrado nestes locais. O comportamento do íon K observado no presente experimento não segue o padrão estabelecido por estes autores.

Os tratamentos de fertirrigação (FI, F1/2, e F1/3) apresentaram maiores teores de $\mathrm{K}$ no solo. $\mathrm{O}$ parcelamento pode ter reduzido a lixiviação deste nutriente, de forma que, ao final do ciclo, ocorreram diferenças. $\mathrm{O}$ contraste entre fertirrigação e aplicação convencional foi significativo a $1 \%$.

O contraste dos tratamentos de fertirrigação e aplicação convencional de fertilizantes, significativo a $4 \%$, aponta uma superioridade nos teores de $\mathrm{P}$ no solo obtidos nos tratamentos de fertirrigação, indicando efeito de parcelamento de doses. No entanto, o momento da última parcela da aplicação de $\mathrm{P}$ foi diferente, sendo que, na aplicação convencional, a última aplicação de MAP ocorreu em fevereiro e na fertirrigação, em junho, aumentando a possibilidade de fixação em função do tempo.

Segundo Papadopoulos (1999), a alta freqüência de fertirrigação fosfatada pode aumentar substancialmente a concentração de fósforo a médio prazo, na solução do solo, acima do esperado pelas considerações sobre a solubilidade do P.

Diferenças significativas entre os valores de matéria orgânica foram observadas para os tratamentos, apontando para um possível efeito da irrigação sobre a velocidade de decomposição da matéria vegetal depositada sobre o solo, uma vez que os restos vegetais roçados nas linhas eram deixados sobre o mesmo. Provavelmente, a matéria orgânica do solo apresentou teores mais elevados no tratamento sem irrigação devido ao solo apresentar baixa retenção de água, gerando menor atividade microbiana e mantendo a concentração da matéria orgânica em relação aos solos irrigados. Os resultados obtidos para matéria orgânica explicam as variações nos valores de CTC encontradas neste experimento.

As variações encontradas para $\mathrm{V} \%$ refletem os teores das bases, principalmente $\mathrm{Ca}$ e $\mathrm{Mg}$.

O contraste entre fertirrigação e aplicação convencional de fertilizantes foi significativo para boro, observando-se valores superiores deste elemento nos tratamentos de fertirrigação. Isto aponta para um efeito do parcelamento, uma vez que o boro é um elemento altamente influenciado pela lixiviação.

\section{Análise foliar}

A análise foliar obtida a partir de folhas coletadas em março de 1999, quando os tratamentos SI e CI, referentes à adubação convencional, já haviam sido concluídos, e, para a fertirrigação, faltavam ainda três aplicações, é apresentada na

TABELA 1 - Valores médios de peso unitário de frutos, números de frutos por planta e produção em t.ha ${ }^{-1}$.

\begin{tabular}{cccc}
\hline Tratamentos & Peso de fruto & $\begin{array}{c}\text { Número de } \\
\text { frutos/planta }\end{array}$ & Produtividade \\
\hline 1 & $\mathrm{G}$ & & t.ha $^{-1}$ \\
2 & 169,4 & 760 & 47,1 \\
3 & 156,0 & 878 & 49,0 \\
4 & 164,8 & 937 & 55,6 \\
5 & 161,8 & 884 & 50,0 \\
\hline Valores F & 161,4 & 746 & 42,4 \\
\hline Pr>F & 0,81 & 1,36 & 0,86 \\
\hline C.V. (\%) & 0,53 & 0,29 & 0,51 \\
\hline
\end{tabular}


TABELA 2 - Médias dos valores de acidez, brix, 'ratio', espessura de casca, rendimento de suco, diâmetro e altura de frutos.

\begin{tabular}{cccccccc}
\hline Tratamentos & Acidez & S S T & 'Ratio' & $\begin{array}{c}\text { Espessura } \\
\text { de casca }\end{array}$ & $\begin{array}{c}\text { Rendimento } \\
\text { de suco }\end{array}$ & $\begin{array}{c}\text { D iâmetro } \\
\text { de fruto }\end{array}$ & $\begin{array}{c}\text { A ltura de } \\
\text { fruto }\end{array}$ \\
\hline & $\%$ & brix & & M m & $\%$ & m m & $\mathrm{mm}$ \\
\hline 1 & 1,97 & 10,47 & 5,35 & 4,28 & 35,24 & 68,14 & 68,57 \\
3 & 1,97 & 10,70 & 5,73 & 3,91 & 34,88 & 65,93 & 65,87 \\
4 & 1,90 & 10,65 & 5,67 & 4,16 & 34,25 & 67,19 & 67,63 \\
5 & 1,88 & 10,18 & 5,48 & 4,03 & 35,03 & 67,04 & 67,98 \\
\hline V a lores F & 2,00 & 10,70 & 5,13 & 4,03 & 35,56 & 66,42 & 67,71 \\
\hline Pr>F & 0,48 & 0,70 & 1,62 & 1,87 & 1,09 & 0,70 & 2,52 \\
\hline C.V.(\% ) & 0,75 & 0,59 & 0,21 & 0,16 & 0,39 & 0,60 & 0,07 \\
\hline
\end{tabular}

TABELA 3 - Média dos valores obtidos pela análise química do solo a $20 \mathrm{~cm}$, em agosto de 1999.

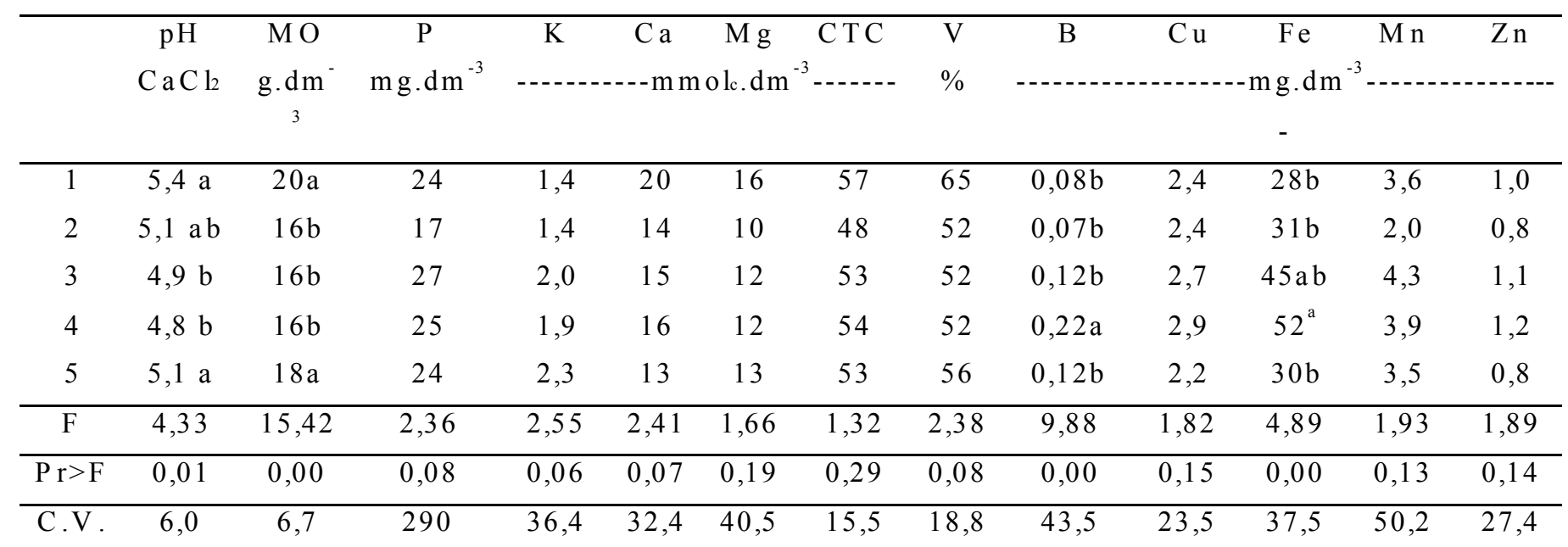

Valores seguidos pela mesma letra não diferem entre si, pelo Teste de Tukey, a 5\%.

TABELA 4 - Médias dos valores obtidos pela análise foliar.

\begin{tabular}{|c|c|c|c|c|c|c|c|c|c|c|c|}
\hline & $\mathrm{N}$ & $P$ & $\bar{K}$ & $\mathrm{Ca}$ & $\mathrm{Mg}$ & $\mathrm{S}$ & B & $\mathrm{Cu}$ & $\mathrm{Fe}$ & $\mathrm{Mn}$ & $\mathrm{Zn}$ \\
\hline 1 & 24,0 & 1,50 & $11,3 \mathrm{ab}$ & $39,3 \mathrm{ab}$ & $5,3 b$ & 2,7 & 120,7 & $59,0 \mathrm{a}$ & 96,3 & 27,0 & 14,7 \\
\hline 2 & 23,7 & 1,50 & $12,3 \mathrm{a}$ & $39,3 \mathrm{ab}$ & $5,8 \mathrm{ab}$ & 2,4 & 153,0 & $47,7 \mathrm{ab}$ & 97,3 & 25,0 & 13,3 \\
\hline 3 & 24,0 & 1,47 & $10,0 \mathrm{~b}$ & $41,3 \mathrm{ab}$ & $6,2 \mathrm{ab}$ & 2,4 & 118,7 & $41,7 \mathrm{ab}$ & 88,3 & 27,3 & 13,3 \\
\hline 4 & 23,3 & 1,57 & $11,0 \mathrm{ab}$ & $37,0 \mathrm{~b}$ & $6,2 \mathrm{ab}$ & 2,5 & 124,3 & $22,7 b$ & 95,0 & 20,3 & 11,3 \\
\hline 5 & 23,7 & 1,53 & $9,7 \mathrm{~b}$ & $43,3 \mathrm{a}$ & $6,8^{\mathrm{a}}$ & 2,3 & 130,0 & $41,0 \mathrm{ab}$ & 82,3 & 22,7 & 14,3 \\
\hline $\mathrm{F}$ & 0,48 & 3,25 & 4,20 & 4,20 & 5,08 & 1,77 & 1,23 & 3,99 & 0,89 & 1,37 & 0,54 \\
\hline C.V. & 2,93 & 2,41 & 5,70 & 5,03 & 7,07 & 9,14 & 16,82 & 26,96 & 12,77 & 17,92 & 22,96 \\
\hline
\end{tabular}

Valores seguidos pela mesma letra não diferem entre si, pelo Teste de Tukey, a 5\%.

Tabela 4.

Diferenças significativas foram observadas apenas para os teores foliares de $\mathrm{K}, \mathrm{Ca}, \mathrm{Mg}$ e $\mathrm{Cu}$.

Os teores de N, P, Ca, S e Fe nas folhas encontram-se dentro da faixa adequada estabelecida por Quaggio et al. (1997). Para K, pode-se observar que apenas o tratamento $\mathrm{F} 1 / 3$, que utiliza a menor dosagem de $\mathrm{K}$, se encontrou abaixo do limite inferior. Para B, todos os tratamentos extrapolaram o limite superior de teor foliar estabelecido como padrão (36 a $100 \mathrm{mg} /$ $\mathrm{kg})$.

Com relação ao $\mathrm{Mg}$, observa-se que todos os tratamentos apresentaram teores altos, superiores ao padrão. 
Deve-se lembrar que os teores de $\mathrm{Mg}$ no solo não obedeceram à proporção entre $\mathrm{Ca}$ e $\mathrm{Mg}$, podendo ter ocorrido absorção em excesso de $\mathrm{Mg}$ com relação ao $\mathrm{Ca}$, uma vez que a redistribuição do $\mathrm{Mg}$ é mais eficiente que a redistribuição do $\mathrm{Ca}$ (Malavolta et al., 2000). Tanto para Ca como para Mg, o tratamento F1/3 foi o que apresentou os teores foliares mais elevados. Este mesmo tratamento apresentou o menor teor de $\mathrm{K}$ observado, sendo que os valores obtidos para estes três elementos, $\mathrm{K}, \mathrm{Ca}$ e $\mathrm{Mg}$, seguem uma tendência: para a maioria dos tratamentos, quando se observam maiores valores de $\mathrm{K}$ nas folhas, os valores de $\mathrm{Ca} e$ $\mathrm{Mg}$ são mais baixos.

Estes resultados estão de acordo com o obtido por Koo et al. (1974), que observaram uma relação inversa nos teores de $\mathrm{K}$ com relação a $\mathrm{Ca}$ e $\mathrm{Mg}$ nas folhas, quando estudaram diferentes doses de $\mathrm{K}$ e $\mathrm{N}$ em limão.

Os teores foliares de $\mathrm{Cu}$ observados em todos os tratamentos são muito elevados, sugerindo um efeito residual de pulverização. Para Mn e Zn, foram observados teores foliares abaixo do padrão, o que aponta para uma possível falha no suprimento destes elementos pelas fontes utilizadas.

\section{CONCLUSÕES}

1. Os tratamentos não afetaram significativamente a produção de frutos.

2. As doses de NPK não produziram diferenças significativas na produtividade da laranja, embora se tenha observado uma tendência de maior produtividade para maiores doses.

3. Os parâmetros de qualidade dos frutos não foram influenciados pelos tratamentos.

4. Os seguintes parâmetros de análise do solo foram influenciados pela irrigação e/ou pelo modo de aplicação dos fertilizantes: $\mathrm{pH}$, matéria orgânica, boro e ferro.

5. Os parâmetros de análise foliar apontaram diferenças entre os tratamentos para teores de $\mathrm{K}, \mathrm{Ca}$ e $\mathrm{Mg}$.

\section{REFERÊNCIAS BIBLIOGRÁFICAS}

ALVA, A.K.; PARAMASIVAM, S. Nitrogen management for high yield and quality of citrus in sandy soils. Soil Science Society of America Journal, Madison, v.62, n.5, p.1335-42, 1998.

ALVA, A.K., PARAMASIVAM, S., GRAHAM, W.D. Impact of nitrogen management practices on nutritional status and yield of Valencia orange trees and groundwater nitrate. Journal of Environmental Quality, Madison, v.27, n.4, p.904-10, 1998.

CASTEL, J.R. Response of young clementine citrus trees to drip irrigation. I. Irrigation amount and number of drippers. Journal of Horticultural Science. Ashford, v.69, n.3, p. 481-89, 1994.

DASBERG, S.; BAR-AKIVA, A., SPAZINSKY, S.; COHEN, A. Fertigation versus broadcasting in an orange grove. Fertilizer Research, Dordrecht, v.15, p. 147-54, 1988.

EMBRAPA. Sistema Brasileiro de Classificação de Solos.
Brasília: EMBRAPA, 1999.412p.

FIGUEIREDO, J.O. Variedades comerciais. In: RODRIGUEZ, O.; VIÉGAS, F.; POMPEU JR., J.; AMARO, A.A. Citricultura Brasileira. 2.ed. Campinas: Fundação Cargill, 1991. v.1, p.22857.

GRUPO PAULISTA DE ADUBAÇÃO E CALAGEM PARA CITROS. Recomendação de adubação e calagem para cítrus no Estado de São Paulo. Laranja, Cordeirópolis, edição especial, 27p, 1994.

GUARDIOLA, J.L. Utilizacion de los reguladores del desarrollo en la produccion de cítricos. In: SIMPÓSIO SOBRE FISIOLOGIA, NUTRIÇÃO, ADUBAÇÃO E MANEJO PARA PRODUÇÃO SUSTENTÁVEL DE CITROS, 2000, Piracicaba. Anais... Piracicaba: POTAFÖS, 2000. CD-ROM.

HAYNES, R.J.; SWIFT, R.S. Effect of trickle fertigation with three forms of nitrogen on soil $\mathrm{pH}$, levels of extractable nutrients below the emitter and plant growth. Plant and Soil, Dordrecht, n.102,p.211-21, 1987.

INTRIGLIOLO, F.; ROCCUZZO, G.; ANAC, D., MARTINPREVEL, P. Evaluation of different fertilization strategies on orange. Improved crop quality by nutrient management, $p$.2326,1999 .

KOO, R.C.J.; YOUNG, T.W.; REESE, R.L.; KESTERTON, J.W. Effects of nitrogen, potassium, and irrigation on yield and quality of lemon. Journal of American Society for Horticultural Science. Alexandria, v.99, n.4, p.289-91, 1974.

MALAVOLTA, E.; MOREIRA, A.; LEÃO, H.C. Nutrição e adubação dos citros - produção, qualidade e doenças. In: SIMPÓSIO SOBRE FISIOLOGIA, NUTRIÇÃO, ADUBAÇÃOE MANEJO PARA PRODUÇÃO SUSTENTÁVEL DE CITROS, 2000, Piracicaba. Anais... Piracicaba: POTAFÖS, 2000. CD-ROM.

PAPADOPOULOS, I. Fertirrigação: situação atual e perspectivas para o futuro. In: FOLEGATTI, M.V. (Coord.) Fertirrigação: cítrus, flores, hortaliças. Guaíba: Agropecuária, 1999. p.11-169.

PREGNOLATTO, W.; PREGNOLATTO, N.P. Normas Analíticas do Instituto Adolfo Lutz: métodos químicos e físicos para análise de alimentos. São Paulo: Instituto Adolfo Lutz, 1985. v.1, 533p.

QUAGGIO, J.A.; RAIJ, B. Van, PIZA JÚNIOR, C.L. Frutíferas. In: RAIJ, B. van, CANTARELLA, H., QUAGGIO, J.A.; FURLANI, A.M.C. (Ed.) Boletim Técnico 100 - Recomendações de adubação e calagem para o Estado de São Paulo, 2.ed. Campinas: IAC, 1997. p.119-52.

TOMLINSON, I.R., COETZEE, K. Can fertigation influence fruit quality? Neltropika Bulletin, n.296, p.7-9, 1997.

TRESSLER, D.K., JOSLYN, M.A. Fruits and vegetables juice processing technology. Westport: AVI, 1961. 1028p. 